\title{
Aligned and Spontaneous Flavor Violation
}

\author{
Daniel Egana-Ugrinovic, ${ }^{1}$ Samuel Homiller, ${ }^{1,2}$ and Patrick Meade ${ }^{1}$ \\ ${ }^{1}$ C. N. Yang Institute for Theoretical Physics, Stony Brook University, Stony Brook, New York 11794, USA \\ ${ }^{2}$ Physics Department, Brookhaven National Laboratory, Upton, New York 11973, USA
}

(Received 26 December 2018; published 17 July 2019)

\begin{abstract}
We present a systematic spurion setup called aligned flavor violation (AFV) that allows for new physics couplings to quarks that are aligned with standard model (SM) Yukawa couplings, but do not necessarily share their hierarchies nor are family universal. Additionally, we show that there is an important subset of AFV called spontaneous flavor violation (SFV), which naturally arises from UV completions where the quark family number and $C P$ groups are spontaneously broken. Flavor-changing neutral currents are strongly suppressed in SFV extensions of the SM. We study SFV from an effective field theory perspective and demonstrate that SFV new physics with significant and preferential couplings to first or second generation quarks may be close to the $\mathrm{TeV}$ scale.
\end{abstract}

DOI: 10.1103/PhysRevLett.123.031802

Introduction.-Strong constraints on flavor-changing neutral currents (FCNCs) and $C P$ violation (CPV) indicate that, naïvely, the scale of new physics needs to be above $\sim 10^{5} \mathrm{TeV}$ [1], beyond the energy frontier (EF) reach of current and future experiments. To reconcile this disparity of scales, assumptions need to be made for the form of any couplings of beyond the SM (BSM) particles to the quarks of the SM, which bias the search for BSM physics. These assumptions can be encoded through EFT and/or spurion methods, the most common being minimal flavor violation (MFV) [2]. MFV imposes that, in extensions of the SM, the breaking of the SM flavor group is entirely from SM Yukawa spurions, so it inherits much of the flavor protection that exists in the SM and allows for new physics at the TeV scale. MFV can be applied to the SM EFT or to specific BSM models and makes precise predictions, the strongest one being that the couplings of new physics to quarks are either flavor blind, as in theories of gauge mediation [3], or retain the hierarchies of the SM Higgs Yukawa couplings. Other extensions of MFV such as GMFV [4] and NMFV [5,6] retain these properties. Thus, while these extensions have EF implications for third-generation BSM searches, they generically provide no more guidance than naturalness considerations.

From the perspective of EF searches it is important to make sure that BSM physics is not missed because of theoretical assumptions. Therefore to extend the scope of flavorful EF searches, we introduce a new flavorful ansatz:

Published by the American Physical Society under the terms of the Creative Commons Attribution 4.0 International license. Further distribution of this work must maintain attribution to the author(s) and the published article's title, journal citation, and DOI. Funded by SCOAP ${ }^{3}$. aligned flavor violation (AFV). AFV allows for phenomenologically interesting couplings to the first and second generation quarks, and/or highly generation-dependent couplings, with the defining property that all flavorchanging processes are CKM suppressed. While the basic concept of "alignment" in flavor physics has long been understood in particular models [7-11], we generalize it to the flavor spurion perspective. This allows one to define AFV theories either as EFTs or as a prescription for how any particular BSM states can couple to quarks as with MFV.

While AFV is an interesting systematic generalization of flavor alignment, it has two important shortcomings. First, CKM suppression is insufficient to reduce the bounds from flavor physics to LHC energies. Second, without a UV completion it is not any more compelling than picking an ad hoc texture for the flavored spurions to suppress FCNCs.

To solve the above issues, we introduce a class of AFV theories that we refer to as spontaneous flavor violation (SFV). In SFV theories the quark family number and $C P$ groups are broken only by wave function renormalization of the SM right-handed up or down quarks (leading to two types of SFV, up type or down type). From the IR perspective, SFV theories restrict the set of allowed AFV spurions, resulting in both CKM and Yukawa suppression of FCNCs. From the UV perspective, the SFV subset is strongly motivated since it is naturally selected by a simple class of UV completions. One example of such UV completions are theories where the quark family number and $C P$ groups are broken spontaneously in some extended flavored sector, and the breaking is communicated to the SM right-handed quarks via mixing with heavy vectorlike quarks. Because these UV completions lead to $C P$ violation only in wave function renormalization, 
they provide a UV solution to the strong $C P$ problem, as Nelson-Barr [12-15] and Hiller-Schmaltz models [16].

In this Letter we demonstrate the basic setup of $\mathrm{AFV}$ and SFV, we derive bounds on SFV theories from an EFT perspective, and we present the example UV completion indicated above for SFV. Just as with MFV or AFV, SFV can be applied to any particular BSM model to determine the couplings to SM quarks, opening up new opportunities for exploring BSM physics at colliders.

Aligned flavor violation.-To define AFV we exploit the symmetries of the SM after EWSB. The quark flavor symmetry group of the $\mathrm{SM}, U(3)_{q}^{3} \equiv U(3)_{Q} \times U(3)_{\bar{u}} \times$ $U(3)_{\bar{d}}$, is broken to $U(1)_{Y} \times U(1)_{B}$ by the background values of the Yukawa spurions

$$
\mathcal{L} \supset-y_{i j}^{u} Q_{i} H \bar{u}_{j}+y_{i j}^{d \dagger} Q_{i} H^{c} \bar{d}_{j} .
$$

The singular value decomposition of the Yukawa matrices is

$$
\begin{aligned}
y^{u} & =U_{Q_{u}} Y^{u} U_{\bar{u}}^{\dagger} \equiv U_{Q_{u}} \operatorname{diag}\left(y_{u}^{\mathrm{SM}}, y_{c}^{\mathrm{SM}}, y_{t}^{\mathrm{SM}}\right) U_{\bar{u}}^{\dagger}, \\
y^{d^{\dagger}} & =U_{Q_{d}} Y^{d} U_{\bar{d}}^{\dagger} \equiv U_{Q_{d}} \operatorname{diag}\left(y_{d}^{\mathrm{SM}}, y_{s}^{\mathrm{SM}}, y_{b}^{\mathrm{SM}}\right) U_{\bar{d}}^{\dagger} .
\end{aligned}
$$

The unitary matrices above transform between a generic flavor basis and the quark mass eigenbasis, where the Yukawa interaction matrices are diagonal. These unitary matrices are defined by Eq. (2) only up to a $U(1)_{R}^{6}=$ $U(1)_{R}^{5} \times U(1)_{B}$ reparametrization group defined by

$$
\begin{aligned}
& U_{Q_{u}(\bar{u})} \rightarrow U_{Q_{u}(\bar{u})} \operatorname{diag}\left(e^{i \alpha_{u}}, e^{i \alpha_{c}}, e^{i \alpha_{t}}\right), \\
& U_{Q_{d}(\bar{d})} \rightarrow U_{Q_{d}(\bar{d})} \operatorname{diag}\left(e^{i \alpha_{d}}, e^{i \alpha_{s}}, e^{i \alpha_{b}}\right) .
\end{aligned}
$$

The $U(1)_{R}^{5}$ factor of the reparametrization group is independent of the flavor group, and can be understood as a symmetry of moving to the mass basis. Physical observables must be flavor and reparametrization invariant. The only nontrivial flavor-invariant combination of the unitary matrices in Eq. (2) is the CKM matrix,

$$
V \equiv U_{Q_{u}}^{T} U_{Q_{d}}^{*}
$$

However, the CKM matrix transforms nontrivially under $U(1)_{R}^{5}$. A choice of $U(1)_{R}^{5}$ basis removes five phases from Eq. (4), leaving the unique physical CKM phase.

In MFV, only tensor products of $y^{u}$ and $y^{d}$ characterize flavor violation. To go beyond this, we introduce new flavored spurions. For instance, we may introduce flavor spurions $\kappa^{u}$ and $\kappa^{d}$, with the same transformation properties under $U(3)_{q}^{3}$ as $y^{u, d}$. In the SM EFT or in a generic BSM theory, this would imply large FCNCs; therefore we require additional restrictions on $\kappa^{u, d}$.

In the SM the CKM matrix is the only source of flavor-changing processes. Going beyond the SM, CKM suppression of flavor-changing effects can be retained imposing the condition that the only flavor invariant spurion that transforms nontrivially under $U(1)_{R}^{5}$ is $V$ itself. This also guarantees that the CKM matrix contains only one physical phase. We define this flavor setup as aligned flavor violation (AFV). All AFV spurions may then be expressed via an alignment expansion in powers of the CKM matrix. As an illustration, the most general AFV spurions $\kappa^{u, d}$ consistent with the flavor and reparametrization symmetries, up to second order in the alignment expansion take the form

$$
\begin{aligned}
\kappa^{u} & =U_{Q_{u}}\left[K^{u}+K^{u^{\prime}} V^{*} K^{u^{\prime}} V^{T} K^{u^{\prime \prime \prime}}+\mathcal{O}\left(V^{4}\right)\right] U_{\bar{u}}^{\dagger}, \\
\kappa^{d^{\dagger}} & =U_{Q_{d}}\left[K^{d}+K^{d^{\prime}} V^{T} K^{d^{\prime \prime}} V^{*} K^{d^{\prime \prime \prime}}+\mathcal{O}\left(V^{4}\right)\right] U_{\bar{d}}^{\dagger},
\end{aligned}
$$

where $K^{x}$ are the matrix coefficients of the alignment expansion and are arbitrary flavor invariant, complexdiagonal $3 \times 3$ matrices. They must be diagonal to ensure that they are $U(1)_{R}^{5}$ invariant, as required by the AFV ansatz. $U(1)_{R}^{5}$ invariance also makes their phases physical $C P$-violating phases. New physics couplings to the three quark generations via the spurions $\kappa^{u, d}$ are neither flavor blind nor respect the hierarchies of the SM Yukawa couplings necessarily, in stark contrast to MFV.

AFV spurions in all $U(1)_{B}$ preserving representations may be obtained by taking tensor products of these basic spurions Eq. (5), and may also be expressed as an expansion in powers of the CKM matrix. Linear combinations and tensor products of AFV spurions are also aligned. In any new physics theory, renormalization group evolution (RGE) only renormalizes the alignment expansion matrix coefficients, so AFV is radiatively stable.

From the UV perspective there is no obvious symmetry reason why AFV should be realized, since the $U(1)_{R}^{5}$ group is only an auxiliary group redefining quark mass eigenstates. In a given model there may be symmetries one can identify to guarantee CKM suppression of FCNCs [8-10,17], but CKM suppression alone is not sufficient to realize new physics at LHC energies. In practice, by symmetries or assumption, models that realize an AFV ansatz select only the lower order terms in the alignment expansion, aiming for simultaneous diagonalizability of the flavor spurions with the SM Yukawa matrices, e.g., Refs. [8,9,11] (for other examples of flavorful theories, see Refs. [18-23]). In the next sections we address these issues by showing that there is a subset of AFV that we refer to as SFV, which has additional suppression beyond CKM and arises from a simple class of UV completions.

Spontaneous flavor violation.-A class of AFV extensions of the SM called SFV is realized if at a UV boundary scale $\Lambda_{\mathrm{BC}}$, flavor-changing processes and $C P$ breaking are introduced exclusively via wave function renormalization of right-handed SM quarks. While there is a straightforward UV completion of this SFV ansatz above $\Lambda_{\mathrm{BC}}$, we first 
show how defining SFV in this way leads to additional suppression of FCNCs beyond CKM suppression in BSM theories, and postpone discussing the UV completion to a later section.

We define an SFV theory by the following two conditions at the boundary scale $\Lambda_{\mathrm{BC}}$ : (1) the only renormalizable interaction breaking the individual quark family number times $C P$ symmetry $U(1)_{f}^{3} \times C P$ is wave function renormalization of either the right-handed up- $(\bar{u})$ or downtype $(\bar{d})$ SM quarks and (2) the theory contains no flavor breaking spurions or fields appearing in renormalizable interactions that transform under $U(3)_{\bar{u}}$ or $U(3)_{\bar{d}}$ correspondingly, besides the SM ones and the wave function matrix above. We refer to theories satisfying the conditions (1) and (2) as up- or down-type SFV depending on the quark being renormalized, regardless of the particular UV completion leading to the first condition. We dedicate the rest of this section to show that in up- (down)-type SFV, all the new flavor spurions are aligned, all down- (up)-type FCNCs are suppressed by factors of SM Yukawa couplings entering in the combinations $V^{T} Y_{u}^{2} V^{*}\left(V^{*} Y_{d}^{2} V^{T}\right)$, and, in addition, new SFV physics may have generic family nonuniversal couplings to SM quarks. Imposing that only wave function renormalization breaks $C P$ is not required for alignment, but ensures that any UV completion leading to the SFV ansatz solves the strong- $C P$ problem [16].

We now demonstrate the features above in up-type SFV. The proof for down-type SFV is obtained by interchanging the up- and down-type quarks in what follows. In up-type $\mathrm{SFV}$, at the scale $\Lambda_{\mathrm{BC}}$, the Lagrangian contains the interactions

$$
\begin{array}{r}
\mathcal{L} \supset i Z_{i j}^{u} \bar{u}_{i}^{\dagger} \bar{\sigma}^{\mu} D_{\mu} \bar{u}_{j}+i \bar{d}_{i}^{\dagger} \bar{\sigma}^{\mu} D_{\mu} \bar{d}_{i}+i Q_{i}^{\dagger} \bar{\sigma}^{\mu} D_{\mu} Q_{i} \\
-\left[\eta_{i j}^{u} Q_{i} H \bar{u}_{j}-\eta_{i j}^{d} Q_{i} H^{c} \bar{d}_{j}+\text { H.c. }\right]+\mathcal{L}_{\mathrm{BSM}},
\end{array}
$$

where without loss of generality we work in the canonical kinetic basis for $\bar{d}$ and $Q$ and we omit other SM interactions without quarks that are not relevant for our discussion. The term $\mathcal{L}_{\mathrm{BSM}}$ represents operators involving any new physics and SM fields, with the defining limitation that no new spurions or fields transforming under $U(3)_{\bar{u}}$ are allowed at the renormalizable level. As a consequence, all renormalizable interactions involving the up-type right-handed quarks contain the product $\eta_{i j}^{u} \bar{u}_{j}$. New physics with renormalizable interactions to the $\mathrm{SM}$ in $\mathcal{L}_{\mathrm{BSM}}$, at a mass scale $\Lambda_{\mathrm{NP}}$, may be accessible at colliders and for instance provide a solution to the hierarchy problem. Nonrenormalizable operators may appear in $\mathcal{L}_{\mathrm{BSM}}$ but are suppressed by mass scales related to the SFV UV completion that are similar to $\Lambda_{\mathrm{BC}}$ or heavier, which are assumed to be inaccessible at current experiments and are neglected in what follows. Finally and also by definition, in Eq. (6) there is a special flavor basis that we commit to, in which $Z_{i j}^{u}$ has off-diagonal complex entries and the matrices $\eta^{u, d}$ are real diagonal, since they respect the $U(1)_{f}^{3} \times C P$ symmetry [i.e., $\eta^{u, d}$ break the flavor group down to $U(1)_{f}^{3} \times C P$, while the wave function matrix further breaks it down to $\left.U(1)_{B}\right]$. Additional allowed spurions in any $U(1)_{B}$ preserving representation of the $U(3)_{Q} \times U(3)_{\bar{d}}$ group that may exist in $\mathcal{L}_{\mathrm{BSM}}$ can be written as tensor products of arbitrary real-diagonal spurions transforming as the down-type SM Yukawa matrix.

To go to the fully canonical kinetic basis, we define the square-root matrix $\sqrt{Z^{u}}$ by

$$
Z^{u}=\sqrt{Z^{u} \dagger} \sqrt{Z^{u}},
$$

and redefine the up-type quarks

$$
\bar{u}_{i}^{\prime}=\left(\sqrt{Z^{u}}\right)_{i j} \bar{u}_{j} .
$$

Dropping the primes on the redefined quarks, the renormalized Lagrangian is

$$
\begin{aligned}
\mathcal{L} \supset & i \bar{u}_{i}^{\dagger} \bar{\sigma}^{\mu} D_{\mu} \bar{u}_{i}+i \bar{d}_{i}^{\dagger} \bar{\sigma}^{\mu} D_{\mu} \bar{d}_{i}+i Q_{i}^{\dagger} \bar{\sigma}^{\mu} D_{\mu} Q_{i} \\
& -\left[y_{i j}^{u} Q_{i} H \bar{u}_{j}-y_{i j}^{d^{\dagger}} Q_{i} H^{c} \bar{d}_{j}+\text { H.c. }\right]+\mathcal{L}_{\mathrm{BSM}},
\end{aligned}
$$

with renormalized Yukawa couplings

$$
\begin{gathered}
y^{u}=\eta^{u}\left(\sqrt{Z^{u}}\right)^{-1}=V^{T} Y^{u}, \\
y^{d \dagger}=\eta^{d}=Y^{d} .
\end{gathered}
$$

The basis in Eqs. (10) and (11) corresponds to the flavor basis obtained by setting $U_{\bar{u}, \bar{d}, Q_{d}}=\mathbb{1}, U_{Q_{u}}=V^{T}$ in Eq. (2). Note that the off-diagonal terms of the CKM matrix are exclusively due to wave function renormalization. The SM down-type Yukawa matrix and all the rest of the spurions appearing in $\mathcal{L}_{\mathrm{BSM}}$ transforming only under $U(3)_{Q} \times$ $U(3)_{\bar{d}}$ are not renormalized, while all SM and BSM interactions involving the up-type right-handed quarks end up containing the product $y_{i j}^{u} \bar{u}_{j}$. As a consequence, in our flavor basis the allowed spurions are the up-type SM Yukawa Eq. (10), the real-diagonal down-type SM Yukawa Eq. (11) and spurions appearing in $\mathcal{L}_{\mathrm{BSM}}$, which can be expressed as tensor products of arbitrary real-diagonal down-type Yukawa matrices. We conclude that the theory is flavor aligned. In particular, SFV only selects the lowest order terms in the alignment expansion, cf., Eq. (5). In addition, since all the down-type quark FCNCs are formed with the bilinears $Q_{i}^{\dagger} Q_{j}, Q_{i} \bar{d}_{j}$, and $\bar{d}_{i}^{\dagger} \bar{d}_{j}$, and since insertions of $y^{u} y^{u^{\dagger}}$ are required in these bilinears to obtain down-type FCNCs, all down-type FCNCs are suppressed by factors of $\left(V^{T} Y_{u}^{2} V^{*}\right)_{i j}$.

In summary, in the up-type SFV ansatz all new spurions transforming under $U(3)_{Q} \times U(3)_{\bar{d}}$ in our flavor basis in Eqs. (10) and (11) are arbitrary $3 \times 3$ real-diagonal matrices 
TABLE I. Selection of dimension-six FCNC operators with their SFV coefficients.

\begin{tabular}{lc}
\hline \hline Operator & SFV factor \\
\hline$\left(Q_{1}^{\dagger} \bar{\sigma}^{\mu} Q_{2}\right)^{2}$ & $C_{D}^{1}=\left(V^{*} K_{d}^{2} V^{T}\right)_{12}^{2} C_{K}^{1}=\left(V^{T} Y_{u}^{2} V^{*}\right)_{12}^{2}$ \\
$\left(Q_{1} \bar{d}_{2}\right)\left(Q_{2}^{\dagger} \bar{d}_{1}^{\dagger}\right)$ & {$\left[\left(V^{T} Y_{u}^{2} V^{*} K^{d}\right)_{12}\left(V^{T} Y_{u}^{2} V^{*} K^{d}\right)_{21}^{*}\right]$} \\
$Q_{2} H^{c} \sigma^{\mu \nu} \bar{d}_{3} F_{\mu \nu}$ & {$\left[\left(V^{T} Y_{u}^{2} V^{*}\right) K^{d}\right]_{23}$} \\
\hline \hline
\end{tabular}

or tensor products of such matrices. No new spurions transforming under $U(3)_{\bar{u}}$ are allowed, so new physics couplings to the right-handed up-type quarks are either flavor blind or formed out of the SM up-type Yukawa matrix. The down-type SFV prescription is similar, but with up- and down-type right-handed quarks interchanged. The $\mathrm{CKM}$ phase is the only $C P$-violating phase in SFV.

SFV is not stable under RGE below the scale $\Lambda_{\mathrm{BC}}$, but RGE corrections are suppressed by both loop and CKM factors in any new physics model, as in Refs. [11,24-26].

Flavor bounds in an example SFV theory.-As an application of the SFV ansatz, consider extending the SM flavor spurion content with only one new down-type Yukawa spurion $\kappa^{d}$. In up-type SFV, $\kappa^{d}$ is guaranteed to be flavor aligned, and in the flavor basis used in the previous section it is also real diagonal,

$$
\kappa^{d^{\dagger}}=K^{d} \equiv \operatorname{diag}\left(\kappa_{d}, \kappa_{s}, \kappa_{b}\right), \quad \kappa_{f} \in \mathbb{R} .
$$

where $\kappa_{d, s, b}$ are arbitrary Yukawa couplings. Such a spurion may for instance couple a second Higgs doublet [27], vectorlike quarks [28] or Z-prime bosons [28-31] to SM quarks.

To assess how effective the SFV ansatz is in suppressing FCNCs for a generic new physics theory, we make use of an EFT approach and explore constraints on dimension six operators. In such an EFT the Wilson coefficients are controlled by products of SM Yukawa matrices and $\kappa^{d}$, as illustrated in Table I. From Table I we see that all FCNCs are suppressed by CKM factors, as expected from any flavor aligned theory, and all down-type FCNCs come with factors of $\left(V^{T} Y_{u}^{2} V^{*}\right)$ as previously stated.

In Table II we present bounds on the scale of the dimension six SFV operators. For comparison, we also show bounds on dimension-six MFV and flavor anarchic operators. Since MFV operators are also allowed by definition in an SFV theory, values of $\kappa_{d, s, b}$ leading to $\Lambda_{\mathrm{NP}}^{\mathrm{SFV}} \leq \Lambda_{\mathrm{NP}}^{\mathrm{MFV}}$ requires us to take the MFV limits instead. Bounds on SFV operators are much weaker than on generic flavor-anarchic new physics. Importantly, the scale at which new SFV physics may be found consistent with flavor bounds is generation specific, since it depends on the three new Yukawa couplings $\kappa_{d, s, b}$ independently. As an example, consider a scenario in which new physics is mostly coupled to first generation quarks. For concreteness, take $\kappa_{s, b}=0$, and $\kappa_{d} \sim 10^{5} y_{d}^{\mathrm{SM}}(\sim 0.1)$. From Table II we
TABLE II. $95 \%$ C.L. bounds on the new physics scale $\Lambda_{\mathrm{NP}}$, for anarchic, SFV, and MFV operator coefficients (from Refs. [1,3234]). Subscripts on the anarchic operator limits indicates that the limit is on the real, imaginary, or absolute value of the operator coefficient.

\begin{tabular}{lccc}
\hline \hline Operator & $\Lambda_{\mathrm{NP}}^{\text {anarchic }}[\mathrm{TeV}]$ & $\Lambda_{\mathrm{NP}}^{\mathrm{SFV}}[\mathrm{TeV}]$ & $\Lambda_{\mathrm{NP}}^{\mathrm{MFV}}[\mathrm{TeV}]$ \\
\hline$\left(Q_{1}^{\dagger} \bar{\sigma}^{\mu} Q_{2}\right)^{2}$ & $1.5 \times 10^{4}{ }_{(\mathrm{Im})}$ & $262.7\left|\kappa_{d}^{2}-\kappa_{s}^{2}\right|$ & 5.1 \\
$\left(Q_{1} \bar{d}_{3}\right)\left(Q_{3}^{\dagger} \bar{d}_{1}^{\dagger}\right)$ & $2.1 \times 10^{3}{ }_{(\mathrm{Abs})}$ & $19.3 \sqrt{\left|\kappa_{d} \kappa_{b}\right|}$ & - \\
$\left(Q_{1} \bar{d}_{2}\right)\left(Q_{2}^{\dagger} \bar{d}_{1}^{\dagger}\right)$ & $2.4 \times 10^{5}{ }_{(\mathrm{Im})}$ & $72.7 \sqrt{\left|\kappa_{d} \kappa_{s}\right|}$ & - \\
$2 e H \sigma^{\mu \nu} Q_{2} \bar{d}_{3} F_{\mu \nu}$ & $276.3_{(\mathrm{Re})}$ & $54.3 \sqrt{\left|\kappa_{b}\right|}$ & 7.0 \\
$2 e H \sigma^{\mu \nu} Q_{3} \bar{d}_{2} F_{\mu \nu}$ & $276.3_{(\mathrm{Re})}$ & $54.3 \sqrt{\left|\kappa_{s}\right|}$ & 7.0 \\
$2 e H \sigma^{\mu \nu} Q_{3} \bar{d}_{1} F_{\mu \nu}$ & $140.5_{(\mathrm{Abs})}$ & $13.2 \sqrt{\left|\kappa_{d}\right|}$ & 7.0 \\
\hline \hline
\end{tabular}

see that new physics with such nonuniversal couplings to first generation quarks may be close to the $\mathrm{TeV}$ scale.

SFV UV completion.-We now present one example UV completion for the up-type SFV ansatz. A UV completion for the down-type SFV ansatz can be trivially obtained with the appropriate up-down replacements. We add to the SM scalar gauge singlets $S_{i A}$ and vectorlike right-handed uptype quarks $U_{A}, \bar{U}_{A}, A=1, \ldots, 3$, where $\bar{U}$ has the same gauge quantum numbers as the SM quark $\bar{u}$. We introduce interactions between the singlets, vectorlike quarks, and uptype right-handed SM quarks. Our Lagrangian is

$$
\begin{aligned}
\mathcal{L} \supset & M_{A B} U_{A} \bar{U}_{B}+\xi S_{i A} \bar{u}_{i} U_{A} \\
& -\left[\eta_{i j}^{u} Q_{i} H \bar{u}_{j}-\eta_{i j}^{d} Q_{i} H^{c} \bar{d}_{j}+\text { H.c. }\right]+\mathcal{L}_{\mathrm{BSM}}
\end{aligned}
$$

where we omit canonical kinetic terms for all fields and other SM interactions without quarks that are not relevant for our discussion. Additional renormalizable interactions to the ones appearing explicitly in Eq. (13) coupling the vectorlike quarks to SM fields may be forbidden by a $\mathbb{Z}_{2}$ symmetry. Without loss of generality, we work in a basis where the vectorlike quark mass matrix is diagonal $M_{A B}=\delta_{A B} M_{A} \cdot \mathcal{L}_{\mathrm{BSM}}$ represents any other interactions involving arbitrary new physics fields and SM fields, with the only constraint that no extra spurions or fields transforming under the SM flavor group factor $U(3)_{\bar{u}}$ appear at the renormalizable level. Next, we impose that $C P$ and SM quark family numbers $U(1)_{f}^{3}$ are good symmetries in the UV. There exists then a flavor basis in which all spurions transforming under the SM flavor group, including the SM Yukawa interactions, are real-diagonal $3 \times 3$ matrices or tensor products of such matrices. We commit to this realdiagonal flavor basis. The Yukawa matrices remain real diagonal under renormalization from the UV, protected by the $U(1)_{f}^{3} \times C P$ symmetries. Finally, we break $U(1)_{f}^{3} \times$ $C P$ softly via a VEV for the singlets. Note that in this theory the strong- $C P$ problem is solved via the Nelson-Barr mechanism since $C P$ violation is introduced only via mixing with vectorlike quarks [12-15]. 


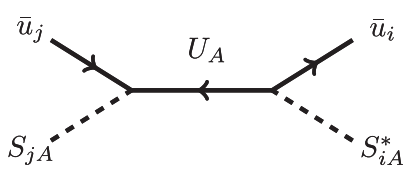

FIG. 1. Diagram leading to the wave function renormalization operator [Eq. (15)].

To understand the effect of the VEV in the infrared, we treat the singlet condensate as a flavor breaking spurion and integrate out the vectorlike quarks. The singlet condensates cannot be $\ll M_{A}$, otherwise in the effective theory flavorchanging processes would be much suppressed and the CKM matrix would be close to the identity. This motivates defining an operator's effective dimension (ED) to be

$$
n_{\mathrm{ED}}=4+n_{M^{2}}-n_{S^{2}},
$$

where $n_{M^{2}}$ and $n_{S^{2}}$ count powers of vectorlike masses and singlet condensate insertions in the operator's coefficient. The leading effects in the infrared are obtained by working up to ED four, higher ED operators have coefficients suppressed by vectorlike quark masses $M_{A}$ and we drop them. In the spurion limit and at tree level, the only contributing diagram to the low energy theory is given in Fig. 1 (plus diagrams related by gauge invariance). At ED four it leads to wave function renormalization of the right-handed up quarks, so the corresponding effective theory is given by Eq. (6), where the up-type quark wave function renormalization matrix is

$$
Z_{i j}^{u}=\delta_{i j}+\frac{\xi^{*} \xi}{M_{A}^{*} M_{A}} S_{i A}^{*} S_{j A}
$$

$Z^{u}$ is not diagonal in quark flavor space, and is the only source of $U(1)_{f}^{3} \times C P$ breaking in the effective theory. We conclude that this theory is a UV completion leading to uptype SFV at the boundary scale $\Lambda_{\mathrm{BC}} \sim M_{A}$.

With this UV completion we do not attempt to provide an explanation for the hierarchies of the SM quark masses, mixing angles and $C P$ phase, which would require a full theory of flavor that is beyond the scope of this Letter. This situation is not any worse than in MFV or other flavor prescriptions, which also require a dynamical explanation of the background values of the flavor spurions [35-40].

Discussion.-AFV and SFV provide a promising generalization of the usually flavor universal ansatz of MFV for BSM physics. Both can be studied from the EFT perspective, but the SFV ansatz is particularly well motivated from UV and IR considerations. We presented one UV completion to the SFV ansatz, but other realizations may be responsible for SFV. For instance, in supersymmetry, nonrenormalization theorems motivate introducing flavor breaking only in wave function renormalization [16,41]. In addition, horizontal Abelian symmetries lead to an approximate realization of AFV [7-9]. Note however, that our UV completion realizes alignment in a different way than horizontal symmetries. This leads to practical differences; e.g., our setup can be applied to theories with one Higgs doublet, differently from Refs. [7,8].

SFV new physics may have large couplings to any quark generation, leading to novel collider and flavor phenomenology. This motivates techniques such as lightquark taggers [42-44] at the LHC and beyond. SFV may be applied to a variety of models, as theories with extra Higgses, vectorlike quarks or $Z$-prime bosons $[27,28,30,31]$. A supersymmetric generalization of the SFV ansatz can be obtained by promoting the SM, vectorlike quark and gauge singlet fields in this Letter to superfields. Another interesting future direction is to embed SFV in a complete theory of flavor.

We would like to thank Luca Di-Luzio, Howard Haber, Duccio Pappadopulo, and Gilad Perez for useful discussions. The work of D. E., S. H., and P. M. was supported in part by the National Science Foundation Grant No. PHY1620628. D. E. and P. M. thank the Galileo Galilei Institute for Theoretical Physics for the hospitality and the INFN for partial support during the completion of this work, as well as support by a grant from the Simons Foundation (Grant No. 341344, LA). P. M. would like to thank the Center for Theoretical Physics at Columbia University for its hospitality during completion of part of this work. S.H. is supported by the Department of Energy SCGSR program, administered by the Oak Ridge Institute for Science and Education which is managed by ORAU under Contract No. DE-SC0014664.

[1] M. Bona et al., Model-independent constraints on $\Delta F=2$ operators and the scale of new physics, J. High Energy Phys. 03 (2008) 049.

[2] G. D’Ambrosio, G. F. Giudice, G. Isidori, and A. Strumia, Minimal flavor violation: An effective field theory approach, Nucl. Phys. B645, 155 (2002).

[3] G. F. Giudice and R. Rattazzi, Theories with gauge mediated supersymmetry breaking, Phys. Rep. 322, 419 (1999).

[4] A. L. Kagan, G. Perez, T. Volansky, and J. Zupan, General minimal flavor violation, Phys. Rev. D 80, 076002 (2009).

[5] K. Agashe, M. Papucci, G. Perez, and D. Pirjol, Next to minimal flavor violation, arXiv:hep-ph/0509117.

[6] Th. Feldmann and Th. Mannel, Minimal flavour violation and beyond, J. High Energy Phys. 02 (2007) 067.

[7] M. Leurer, Y. Nir, and N. Seiberg, Mass matrix models, Nucl. Phys. B398, 319 (1993).

[8] Y. Nir and N. Seiberg, Should squarks be degenerate, Phys. Lett. B 309, 337 (1993).

[9] M. Leurer, Y. Nir, and N. Seiberg, Mass matrix models: The Sequel, Nucl. Phys. B420, 468 (1994).

[10] G. C. Branco, W. Grimus, and L. Lavoura, Relating the scalar flavor changing neutral couplings to the CKM matrix, Phys. Lett. B 380, 119 (1996). 
[11] A. Peuelas and A. Pich, Flavour alignment in multi-Higgsdoublet models, J. High Energy Phys. 12 (2017) 084.

[12] A. E. Nelson, Naturally weak $C P$ violation, Phys. Lett. 136B, 387 (1984).

[13] S. M. Barr, Solving the Strong CP Problem Without the Peccei-Quinn Symmetry, Phys. Rev. Lett. 53, 329 (1984).

[14] S. M. Barr, A natural class of nonpeccei-quinn models, Phys. Rev. D 30, 1805 (1984).

[15] L. Bento, G. C. Branco, and P. A. Parada, A Minimal model with natural suppression of strong $C P$ violation, Phys. Lett. B 267, 95 (1991).

[16] G. Hiller and M. Schmaltz, Solving the strong $C P$ problem with supersymmetry, Phys. Lett. B 514, 263 (2001).

[17] A. Antaramian, L. J. Hall, and A. Rasin, Flavor Changing Interactions Mediated by Scalars at the Weak Scale, Phys. Rev. Lett. 69, 1871 (1992).

[18] T. P. Cheng and M. Sher, Mass matrix Ansatz and flavor nonconservation in models with multiple Higgs doublets, Phys. Rev. D 35, 3484 (1987).

[19] S. Bar-Shalom and A. Rajaraman, Models and phenomenology of maximal flavor violation, Phys. Rev. D 77, 095011 (2008).

[20] G. F. Giudice, B. Gripaios, and R. Sundrum, Flavourful production at hadron colliders, J. High Energy Phys. 08 (2011) 055.

[21] B. Batell, A. Freitas, A. Ismail, and D. Mckeen, Flavorspecific scalar mediators, Phys. Rev. D 98, 055026 (2018).

[22] S. Bar-Shalom and A. Soni, Universally enhanced lightquarks Yukawa couplings paradigm, Phys. Rev. D 98, 055001 (2018).

[23] B. Batell, A. Freitas, A. Ismail, and D. McKeen, Probing light dark matter with a hadrophilic scalar mediator, arXiv: 1812.05103 .

[24] S. Gori, H. E. Haber, and E. Santos, High scale flavor alignment in two-Higgs doublet models and its phenomenology, J. High Energy Phys. 06 (2017) 110.

[25] F. J. Botella, F. Cornet-Gomez, and M. Nebot, Flavour conservation in two Higgs doublet models, Phys. Rev. D 98, 035046 (2018).

[26] M. Dine and P. Draper, Challenges for the Nelson-Barr mechanism, J. High Energy Phys. 08 (2015) 132.

[27] S. Davidson and H. E. Haber, Basis-independent methods for the two-Higgs-doublet model, Phys. Rev. D 72, 035004 (2005); Erratum, Phys. Rev. D 72, 099902(E) (2005).
[28] B. Grinstein, M. Redi, and G. Villadoro, Low scale flavor gauge symmetries, J. High Energy Phys. 11 (2010) 067.

[29] P. Langacker and M. Plumacher, Flavor changing effects in theories with a heavy $Z^{\prime}$ boson with family nonuniversal couplings, Phys. Rev. D 62, 013006 (2000).

[30] P. J. Fox, J. Liu, D. Tucker-Smith, and N. Weiner, An effective Z', Phys. Rev. D 84, 115006 (2011).

[31] C. D. Carone, Flavor-nonuniversal dark gauge bosons and the muon g-2, Phys. Lett. B 721, 118 (2013).

[32] M. Bona et al., Constraints on New Physics from the Quark Mixing Unitarity Triangle, Phys. Rev. Lett. 97, 151803 (2006).

[33] A. Crivellin and L. Mercolli, $B \rightarrow X_{d} \gamma$ and constraints on new physics, Phys. Rev. D 84, 114005 (2011).

[34] W. Altmannshofer and D. M. Straub, New physics in $b \rightarrow s$ transitions after LHC run 1, Eur. Phys. J. C 75, 382 (2015).

[35] E. Nardi, Naturally large Yukawa hierarchies, Phys. Rev. D 84, 036008 (2011).

[36] J. R. Espinosa, C. S. Fong, and E. Nardi, Yukawa hierarchies from spontaneous breaking of the $S U(3)_{L} \times S U(3)_{R}$ flavour symmetry, J. High Energy Phys. 02 (2013) 137.

[37] C. S. Fong and E. Nardi, Quark masses, mixings, and $C P$ violation from spontaneous breaking of flavor $S U(3)^{3}$, Phys. Rev. D 89, 036008 (2014).

[38] T. Feldmann, M. Jung, and T. Mannel, Sequential flavour symmetry breaking, Phys. Rev. D 80, 033003 (2009).

[39] M. E. Albrecht, Th. Feldmann, and Th. Mannel, Goldstone Bosons in effective theories with spontaneously broken flavour symmetry, J. High Energy Phys. 10 (2010) 089.

[40] S. Davidson, G. Isidori, and S. Uhlig, Solving the flavour problem with hierarchical fermion wave functions, Phys. Lett. B 663, 73 (2008).

[41] G. Hiller and M. Schmaltz, Strong weak $C P$ hierarchy from nonrenormalization theorems, Phys. Rev. D 65, 096009 (2002).

[42] M. Aaboud et al., Search for the Decay of the Higgs Boson to Charm Quarks with the ATLAS Experiment, Phys. Rev. Lett. 120, 211802 (2018).

[43] A. M. Sirunyan et al., Identification of heavy-flavour jets with the CMS detector in pp collisions at $13 \mathrm{TeV}$, J. Instrum. 13, P05011 (2018).

[44] R. Aaij et al., Identification of beauty and charm quark jets at LHCb, J. Instrum. 10, P06013 (2015). 